\title{
Vom Hin und Her zwischen Medienbildung und Informatischer Bildung
}

\author{
Ein Versuch einer systematischen Verbindung für die Schulpraxis \\ David Stadler ${ }^{1}$ (D) und Nina Grünberger ${ }^{1}$ (D) \\ ${ }^{1}$ Pädagogische Hochschule Wien
}

\begin{abstract}
Zusammenfassung
Informatik hier, Medienpädagogik dort. Informatische Bildung hier, Medienbildung dort. Der Diskurs um digitale Medien in der Bildung zeigt eine duale Aufspaltung bzw. zwei parallel verlaufende, teils schwer verbindbare Aktionsfelder, die von jeweils unterschiedlichen Akteurinnen und Akteuren bedient werden. Eine solche Dualität könnte bspw. auf die jeweiligen Herkunftsdisziplinen, der Informatik bzw. Pädagogik, Kommunikations- und Medienwissenschaft, zurückgeführt werden, wodurch sich widersprechende Logiken und Ziele sowie letztlich eine problematische zweigleisige Koexistenz ergeben. Dabei beanspruchen die Disziplinen die thematische Vorherrschaft gegenüber den anderen. Aktuell scheint etwa die stärkere Thematisierung des Begriffs «Computational Thinking» zulasten der Medienbildung bzw. Medienpädagogik zu gehen. Der Beitrag diskutiert das Beziehungsverhältnis von Informatischer Bildung und Medienbildung entlang einer Darlegung möglicher Gemeinsamkeiten und Unterschiede. Im Anschluss werden anhand der Verortung von Anliegen beider Diskurse in der pädagogischen Praxis Möglichkeiten und Grenzen der konzeptuellen Verbindung der Informatischen Bildung mit der Medienbildung ausgelotet. Dies mündet in einer kritischen Einordnung des Lehrplans der Verbindlichen Übung «Digitale Grundbildung» für österreichische Schulen der Sekundarstufe I, der Ansätze einer Verzahnung von Medienbildung und Informatischer Bildung erkennen lässt. Leitend ist dabei die These, dass die Medienbildung sich der Informatik und diese sich der Medienbildung öffnen solle, um den Anforderungen einer digital-vernetzten Lebenswelt gerecht zu werden.
\end{abstract}

The Conflict of Media Education and Computer Science Education in the German Speaking Discourse

\begin{abstract}
In the German speaking discourse of education, media, IKT, digital skills e.g., there is a huge gap between concerns of computer science and media education. Based on rather politic initiatives as well as of the engagement from a more technical perspective the term
\end{abstract}


"digital education» (German: "Digitale Bildung" or "Digitale Grundbildung") is more and more present. In the scientific discourse of education and particularly in media education the term "Medienbildung" and "Medienkompetenzen" (similar to "media literacy") are used. It seems that these two research fields pursue similar goals and serve fields of action and yet show insurmountable differences. A possible reason for this contradiction lies in their origins in different disciplines: computer science on the one and the pedagogic and media science on the other hand. This article aims to discuss the conceptual relationship between computer science education and media education after explaining possible similarities and differences of the concepts. In the context of Computational Thinking, a critical look is taken at the curriculum for the mandatory exercise "digital education" in Austrian secondary schools, which reveals approaches to interleave media education and informatics. The guiding principle is always the thesis that media education should open up to computer science and that computer science should open up to media education.

\section{Einleitung}

Die Rede von der «Digitalisierung» oder «Mediatisierung» im Generellen und im Speziellen im Bildungsdiskurs beschreibt meist einen Leitmedienwechsel. Das Potenzial, eine derart grosse Umwälzung für das Menschsein anzustossen, hat bereits Marshall McLuhan (1962) in seiner «Gutenberg-Galaxis» der Erfindung des Buchdrucks zugesprochen. Die von Gutenbergs Erfindung ausgelöste Transformation bezog sich auf sämtliche sozio-kulturelle Strukturen. Ähnliche Dimensionen nimmt auch das Transformationspotenzial von «Digitalisierung» bzw. «Mediatisierung» ein (vgl. bspw. Krotz 2001; zur Begriffsbestimmung vgl. bspw. Bettinger und Aßmann 2017), welche gar neue Kulturtechniken einfordert (Meder 2004). Da liegt es auf der Hand, dass dahingehend auch Bildungs- und Erziehungsfragen im Allgemeinen und die Frage der Gestaltung von Schule im Besonderen neu gestellt werden. Beat Döbeli Honegger $(2017,41)$ macht die Veränderung von Bildung und Bildungseinrichtungen wie Schulen an fünf Dimensionen fest: Zum Ersten würden sich Sozialisationsprozesse von Kindern und Jugendlichen durch die Ubiquität digitaler Medien gegenwärtig anders darstellen. Zum Zweiten verändere sich das Berufsleben und damit auch Fragen der Berufsbildung. Zum Dritten evoziere die Digitalisierung einen «Verlust des schulischen Informationsmonopols». Dies kann in Verbindung zu Jean-Francois Lyotards (1979) Verlust der Legitimation von Wissen und Zunahme an Widerstreitigkeiten in der Postmoderne gelesen werden. Zum Vierten kommen «neue Werkzeuge für das Lernen und Arbeiten» auf und zum Fünften bedarf es eines «neue[n] Verständnis[ses] der heutigen Welt», da die gegenwärtige Welt anders funktioniert. Um sozio-kulturelle, ökonomische und ökologische Prozesse und Tendenzen nachvollziehen zu können, bedarf es eines «Grundverständnisses des Digitalen» und der Digitalität. Schulentwicklungsprozesse müssen die «Relationierung von Schule in einer Kultur der Digitalität reflektieren» (Schiefner-Rohs 2017, 153). 
Wir leben in einer Zeit der Schnelllebigkeit, des Widerstreits und von Unsicherheiten. Diese rühren zum Teil wohl auch aus der stets zunehmenden Geschwindigkeit technologischer Entwicklungen (etwa seit Anfang der 1980er Jahre), die mitunter die Existenz ganzer Berufsgruppen scheinbar bedroht. Den jüngeren Generationen wird zugesprochen, diesen schnelllebigen Veränderungen gelassener entgegenzublicken oder disruptive Technologie gar «schick» zu finden (von Weizsäcker und Wijkman 2018, 101). Schliesslich seien sie in der digital-vernetzten Welt aufgewachsen und in der Lage, ihr Verhalten im Einklang mit neuen technischen Innovationen, E-Commerce-Lösungen und Apps situationsadäquat in kürzester Zeit anzupassen.

Die Grundlage für die heute bestehende globale digitale Vernetzung kann mit dem Aufkommen der (kommerziellen) Informations- und Kommunikationstechnik (IKT) angegeben werden, als die ersten Personal Computer auf den Markt kamen und Fernsprechnetze digitalisiert wurden. Digitalisierungsprozesse durchdringen ab diesem Zeitpunkt zunehmend die Lebenswelt der Menschen; die Geschwindigkeit technologischer Entwicklung nimmt stets zu. Die Durchdringung der Lebenswelt und des Alltags durch digitale Technologie macht eine Ko-Evolution des Menschen, parallel mit der Entwicklung seiner technologischen Infrastruktur, nötig; wie dies Manfred Faßler (2009) für alle Ebenen des Menschseins - von der anthropologischen bis zur sozialen Dimension - konstatiert.

Diese Durchdringung des menschlichen Lebensraums und der Lebensgestaltung ist schliesslich Auslöserin genereller Fragen, die in der Pädagogik diskutiert werden. So betont etwa Wolfgang Klafki Ende der 1990er, dass es einer zukunftsbezogenen Allgemeinbildung bedarf, die darauf abzielt «Menschen anzuregen und sie dabei zu fördern, erkenntnisfähig, sensibel, d. h. mitempfindungsfähig, urteilsfähig und handlungsfähig für ihre Gegenwart und Zukunft zu werden» (Klafki 1996, 9). Als «epochaltypische Schlüsselprobleme» nennt Klafki (1998) solche Probleme, die eine enorme gesellschaftliche Herausforderung darstellen und - aus heutiger Sicht - unmittelbar mit Digitalisierungsprozessen verbunden sind: Friedensfragen, Umweltprobleme, Gefahren und Möglichkeiten der neuen technischen Steuerungs-, Informations-, und Kommunikationsmedien im Hinblick auf die Weiterentwicklung des Produktionssystems, der Arbeitsteilung, Vernichtung von Arbeitsplätzen durch Rationalisierung usw. - Digitale Technologien sind dabei sowohl Ursache als auch Lösung dieser Problemlagen. Gegenwärtig gilt, dass wir die Digitalisierung «ausdrücklich in den Dienst der Nachhaltigkeit» stellen müssen (WBGU 2019, 7). Dabei geht es nicht darum, einen Zugang zu entwickeln, der, wie der Medienpädagogik attestiert wird, einer «Reaktion auf die jeweils «neuen Medien»» (Hüther und Podehl 2005, 267) gleicht. Es geht darum, mit Technologie Modelle und Visionen einer nachhaltigen Zukunft zu entwickeln. 
Ziel der Medienbildung ist die Begleitung von Kindern und Jugendlichen zu einem sicheren, selbstreflexiven, kritischen und kreativ-gestalterischen Medienhandeln. Ziel der Informatischen Bildung ${ }^{1}$ ist - etwas verkürzt - die Förderung des Verstehens von informatischen Systemen zur aktiven Mitgestaltung dieser sowie als Möglichkeit einer Problemlösungsstrategie. Beide diese Anliegen lassen sich letztlich für das gemeinsame Ziel zusammenführen. Doch obwohl die Ziele gar so ähnlich klingen, gibt es eine Art Richtungsstreit, der sich durch Verwendung des Wortpaars Medienbildung und Informatische Bildung beispielsweise im wissenschaftlichen Diskurs, in Studiengangbezeichnungen an Hochschulen und in Lehrplänen für Schulen widerspiegelt.

Der vorliegende Beitrag widmet sich dem Beziehungsverhältnis dieser beiden Begriffe und der dahinterliegenden Konzepte und versucht im Anschluss eine Versöhnung dieser beiden vorzunehmen, die sich an gemeinsamen Zielen zum Einsatz in der schulischen Praxis orientiert. Dabei wird insbesondere der Lehrplan zur Verbindlichen Übung «Digitale Grundbildung» (BKA 2018, 14) fokussiert, der mit dem Schuljahr 2018/19 an Mittelschulen und an Allgemeinbildenden Höheren Schulen der Unterstufe in Österreich eingeführt wurde.

\section{Divergierende Perspektiven und Argumentationen?}

Ein genauerer Blick auf das Verhältnis von Medienbildung und Informatischer Bildung eröffnet drei Perspektiven, die meist die argumentative Grundlage diskursiver, zum Teil divergierender Verhandlungen ist.

Dies ist erstens das Argument der (1) Lebensweltorientierung: Es ist das Ziel, Menschen jeden Alters ein sicheres, selbstreflexives und auf allen Ebenen gesundes Leben in einer digital-vernetzten Welt zu ermöglichen. Dazu finden sich unterschiedliche Konzepte, die ebenso unterschiedliche notwendige Aspekte für ein solches Leben formulieren. Es zeigt sich eine Ausdifferenzierung in einerseits allgemeinen Bildungsfragen á la Kant: Was kann ich in einer digital-vernetzen Welt wissen und was nicht? Was soll ich tun oder was soll ich eher nicht tun? Was darf ich hoffen oder was scheint unmöglich? Was ist der Mensch und was ist der Mensch etwa im Verhältnis zu Computern oder Robotern (vgl. dazu bspw. Faßler 2009)? Und es zeigen sich andererseits marktwirtschaftlich-orientierte Fragen, die ebenso an Kants Fragen orientiert werden können: Was muss ich für eine «digitalisiertes Berufswelt wissen? Welche Berufe kann ich zukünftig ausüben? Auf eine wie geartete Berufswelt darf ich hoffen? Was ist der Mensch in einer digitalisierten Arbeitswelt? Für beide Ausdifferenzierungen - also die allgemeinbildende und die marktwirtschaftlich orientierte - ist

1 Neben einer ‘Informatischen Bildung) findet sich ebenso häufig die Reden von einer «Informatischen Grundbildung, welche insbesondere im Kontext der Elementarpädagogik und Primarstufe an Bedeutung zunimmt. Dabei verweist das Präfix Grund- darauf, dass es um eine erste Annäherung an informatische Inhalte gehe. Im vorliegenden Beitrag wird von einer Unterscheidung zwischen «Informatischer Bildung) und ¿Informatischer Grundbildung` abgesehen. 
ein «Verständnis der zugrunde liegenden Technologien und Konzepte» notwendig und ermöglicht das «Verstehen und Mitgestalten zukünftiger Entwicklungen» (Döbeli Honegger 2017, 69). Eine Lebensweltorientierung, die mehr als die Berufswelt und neben Vergangenheit und Gegenwart auch Zukunftsfragen in den Blick nimmt, wird häufig im Kontext des Medienbildungsdiskurses thematisiert (vgl. bspw. Iske 2015). Dies erfolgt unter der Betonung der fruchtbaren Zusammenführung des Begriffspaares Medien und eines allgemein-bildungswissenschaftlichen Verständnisses von Bildung.

Im Kontext der Lebensweltorientierung hat sich gezeigt, dass zweitens eine stärker (2) marktwirtschaftliche Position eingenommen wird, die nicht selten neoliberalen Zielen aufsitzt. In diesem Zusammenhang geht es eher um Fragen nach Bedarfen spezifischer Kompetenzen der erwerbstätigen Bevölkerungsschicht in einem möglichen zukünftigen ‘digitalisierten` Arbeitsmarkt und um Ideen und Visionen zur Förderung eines «digitalen Wirtschaftsstandorts» und der Etablierung neuer «Geschäfts- und Arbeitsmodelle» (Bundesministerium für Digitalisierung und Wirtschaftsstandort 2016). Dieser Bereich ist stärker von politischen Zerrspielen beeinflusst und durch eine eigene, $d$. h. eine andere Begriffsverwendung als etwa in erziehungswissenschaftlich-orientierten Diskursen gekennzeichnet. So findet sich etwa die stark kritisierte Formel der «digitalen Bildung»- Bildung als Prozess kann nicht digital sein - und des «Mehrwerts» digitaler Technologie - digitale Technologie ist ein Werkzeug unter vielen und bringt nicht per se einen nicht näher spezifizierten (Mehrwert) mit (Döbeli Honegger 2017, 68). Daran gekoppelt ist ein «Effizienzargument», wonach digitale Medien gewisse Abläufe effizienter gestalten lassen (Döbeli Honegger 2017, 71). In diesem Kontext geht es selten um Medienbildung oder um andere, einer Allgemeinen Bildungswissenschaft zuordenbare, Grundprinzipien. Die Rede ist dabei häufiger von Digitaler Bildung, Digitalen Kompetenzen oder auch Digital Skills. Ob diese eher unpräzise Begriffsverwendung dem eigentlichen Konzept der Informatischen Bildung gerecht wird, wird noch gezeigt werden.

Wie bereits angeklungen ist, wird der Diskurs von unterschiedlichen wissenschaftlichen Disziplinen geführt. Dabei finden sich selten inter- oder transdisziplinäre Zugänge, wie häufig gefordert (vgl. bpsw. Grünberger 2017). Es zeigen sich verstärkt Diskurse mit einer starken (3) Orientierung an der jeweiligen Herkunftsdisziplin. Als Leitdisziplin der Medienbildung fungiert die Medienpädagogik mit ihren Ursprüngen in den Erziehungs-, Kommunikations- und teilweise Medienwissenschaften (vgl. bpsw. Moser 2006; Schorb 2011). Demgegenüber steht die Informatik als Kerndisziplin der Informatischen Bildung. Beide disziplinären Zugänge haben ihre je eigenen historisch gewachsenen Logiken, Sprachspiele und Methodologien. Die disziplinären Unterschiede werden durchaus auch zum Markieren jeweiliger Relevanzen in Forschungskontexten zum Ziel der Sicherung von Fördertöpfen betont. 
Der Medienbildungsdiskurs wird also vorrangig durch die Bildungswissenschaften mit Seitensträngen aus den Kommunikations- und Medienwissenschaften geführt (vgl. bspw. Trültzsch-Wijnen 2017). Dabei hat sich der Diskurs um Medienbildung erst in den letzten Jahren intensiviert und sukzessive vom Medienkompetenzdiskurs distanziert (gut skizziert bspw. in Fromme und Jörissen 2010). Diese Ablösung folgt - verkürzt formuliert - zwei Leitgedanken: einerseits, dass Zukunftsfragen durch Digitalisierung bzw. Mediatisierung so weitreichend sind, dass ihnen mit heute benennbaren Kompetenzen nicht entsprochen werden kann; und andererseits, dass jede Bildung im Kontext einer digital-vernetzten Welt Medienbildung oder jede Medienbildung im Grunde Bildung sei. Insofern könne generell von «Medienbildung» gesprochen werden oder aber das Präfix «Medien-» weggelassen werden. Jedenfalls gehe es um Fragen von Selbst- und Weltverhältnis im Kontext einer digital-vernetzten Welt. In beiden Varianten werden jedoch Anliegen der Informatischen Bildung nicht benannt. Um dem Rechnung zu tragen wird häufig der Begriff «digitale Bildung» bemüht, wobei hier vehementer Gegenwind von Seiten der Pädagogik kommt, nämlich insofern, als «Bildung» vom Subjekt ausgehe und maximal angeregt werden kann, aber niemals «digital» sei. Das Problem dabei ist, dass sich «Medienbildung〉 konzeptuell-programmatisch sowie politisch, wirtschaftlich schwer verkaufen lässt, wie Bildung eben schwer auf handhabbare Kataloge, knapp formulierte Slogans oder Kennzahlen reduziert werden kann. Gerade der Versuch der Reduktion und Vereinfachung widerspricht einem umfassenden, kritischen Medienbildungsbegriff. Um jeden Preis gilt es Bildung vor neoliberalen Effizienzlogiken und -rhetoriken zu bewahren. So ist es logisch, dass der Digitalen Bildung aus Medienbildungsperspektive fehlender Weitblick und ein mangelndes Verständnis des strukturellen Zusammenhangs von Individuum, Sozialität, Lebenswelt und Informatik attestiert wird. So wird sowohl in einer Stellungnahme der GMK (Gesellschaft für Medienpädagogik und Kommunikationskultur) (2016) als auch in einer Stellungnahme der GI (Gesellschaft für Informatik) (Brinda et al. 2016, 11) die Sorge in den Raum gestellt, dass durch den einseitig gesetzten Fokus auf die Digitalisierung individuelle und soziale bzw. gesellschaftliche/ kulturelle Einflüsse nicht ausreichend mitgedacht werden. Informatische Inhalte seien zwar wesentlich, deren Vermittlung solle aber vorrangig über eine (informations-) technologische Perspektive erfolgen (Dengel 2018, 16).

Die Informatik hat ihrerseits als Disziplin wenig mit Bildung und Erziehung zu tun, es sei denn es geht um das Fach Informatik und damit um die Fachdidaktik Informatik. Dabei orientiert sich der Diskurs konzeptuell häufig am Dagstuhl-Dreieck (2016) sowie am darauf aufbauenden Frankfurter Dreieck (2019). Die sich daraus ergebende - hier etwas verkürzte - Argumentation ist jene, dass die Allgemeinbildung eben auch eine «Digitale Bildung» bräuchte, die in der «Verknüpfung von Inhalten und Kompetenzen der Informatik und Medienbildung» bestünde (Dengel 2018, 19). Der Orientierung an den oben genannten Dreiecken folgt einer Orientierung an drei Aspekten 
nämlich einer technologischen, gesellschaftlich-kulturellen und anwendungsbezogenen Perspektive. Wenngleich die Informatik oft der Reduktion der Inhalte und des fehlenden Weitblicks bezichtigt wird, zeigt sich zumindest hier ein holistisches Anliegen eingedenk sozio-kultureller Bedingungen. Die technologische Perspektive schliesst neben «Wirkprinzipien, Erweiterungs- und Gestaltungsmöglichkeiten [der] Systeme» auch «die Vermittlung grundlegender Problemlösestrategien und -methoden» und des technologischen «Hintergrundwissen[s] für eine Mitgestaltung der digitalen vernetzten Welt» ein (Dengel 2018, 19).

Die Unterschiede der Positionen führen dazu, dass jede für sich die Vormachtstellung einfordert, um Ressourcen kämpft und politische Strategien auf Vorhandensein der eigenen Kernargumente abgeklopft werden. So kann bspw. für die vielzitierte Strategie der Deutschen Kultusministerkonferenz mit dem Titel «Bildung in der digitalen Welt» (2016) nachgewiesen werden, dass der Begriff der Informatik «keinen Einzug gefunden hat und kein einziges Mal erwähnt wird» (Dengel 2018, 15) und gleichzeitig Medienpädagoginnen und Medienpädagogen die starke Orientierung an informationstechnologischen Gesichtspunkten kritisieren. So komme die Informatik im Rahmen der KMK-Strategie zu kurz, bei gleichzeitigem «starken Fokus auf Information und Medien»:

«Digitale Bildung umfasst die Suche, Verarbeitung, Speicherung und Kommunikation von Information sowie die Produktion, Präsentation, Analyse und Reflexion von Medienprodukten. In einer sozialen Komponente beinhaltet sie Kommunikation, Problemlösefähigkeit und Handeln im digitalen Raum. Eine Dreiteilung der digitalen Bildung würde somit Informationen, Medien und soziale Interaktion im digitalen Raum umfassen» (Dengel 2018, 19).

Die KMK-Strategie für Deutschland kann aus österreichischer Perspektive auch als Mahnmal gelesen werden; denn in Österreich gibt es bis dato kein vergleichbares politisches Dokument, dass sich zur Frage Medienbildung und/oder/versus Informatischer Bildung im Bildungskontext positioniert. Eine Gelegenheit für die Konzeption eines derartigen Dokuments war der Diskurs zur Adaptierung einiger Lehrpläne für Schulen in Österreich. Dabei gab es erste Verknüpfungsversuche von Inhalten der Medienbildung mit der Informatik. Ein genauerer Blick in beispielsweise dem verordneten Lehrplan der Verbindlichen Übung «Digitale Grundbildung» verdeutlicht das Entwicklungspotential dieser fruchtbaren Verknüpfung (siehe Kapitel 5).

Weitere Bemühungen um eine systematische Verbindung der Informatischen Bildung mit der Medienbildung sind somit naheliegend, wenngleich dabei neben möglichen Gemeinsamkeiten ebenso Herausforderungen aufgezeigt werden, die in den genannten immanenten Eigenheiten der beiden Disziplinen fussen. 
3. Medienbildung und Informatische Bildung konvergierend gedacht?

Medienbildung und Informatische Bildung sprechen also gewissermassen unterschiedliche Sprachen, die nicht eklektizistisch verknüpft werden können. Anders formuliert: Eine systematische Verbindung der Grundprinzipien der beiden Disziplinen scheint unmöglich. Möglich ist allerdings, deren in den Diskursen verhandelte Beziehungsverhältnisse näher zu beschreiben. So könnte man hinsichtlich des Verhältnisses von Medienbildung und Informatischer Bildung

a. gewissermassen eine Berührungslosigkeit attestieren;

b. eine Schnittmenge im Bildungskontext in Form eines ähnlichen Zieles zusprechen;

c. zuerkennen, Medienbildung sei Teil Informatischer Bildung;

d. zuerkennen, Informatische Bildung sei Teil der Medienbildung.

Doch nimmt man die Medienbildung und Informatische Bildung in ihren Grundprinzipien und programmatischen Zielen der Bildung für eine und in einer digitalvernetzten Welt einschliesslich offener Zukunftsfragen ernst, dann kann keiner der beiden Zugänge negiert werden. Beide sollten sich ihre Spezifika bewahren und für die Sache und in der Sache zusammengeführt werden. Medienbildung und Informatische Bildung oder Medienbildungsfragen und Fragen der Informatik sollen mit ihrem jeweiligen Pouvoir am Diskurs um Zukunftsfragen beteiligt sein.

Weil in der pädagogischen Praxis die «Verzahnung von Informatikdidaktik mit Medienpädagogik und Schulpädagogik» (Schmid und Gärtig-Daugs 2018, 78) häufig gefordert wird, soll dieser Zielsetzung in folgendem Abschnitt Aufmerksamkeit geschenkt werden.

\section{Verbindung Informatischer Bildung und Medienbildung in der pädagogischen Praxis}

Ziel des Versuchs der konzeptuellen Zusammenführung von Medienbildung und Informatischer Bildung im Schulkontext ist die Ermöglichung einer "proaktiven und souveränen Lebensgestaltung von Individuen im Umgang mit Medien, Informationen und Phänomenen in der medialen, technischen und vernetzten Welt» (Dengel 2018, 23). Aus dieser Zielformulierung ergibt sich, dass eine rein technologische Perspektive erweitert werden muss, nämlich um eine strukturelle Perspektive von Digitalität der Gegenwart bezogen auf das Individuum, Sozialitäten und Lebenswelt; um das Zusammenspiel von Gesellschaft und aktiv teilhabendes Individuum in einer digitalvernetzten Welt unter dem Vorzeichen des sozialen Gemeinwohls; um Fragen der «anwendungsbezogenen und wissensbezogenen Handhabe, Auswahl und Begründung digitaler Werkzeuge zur individuellen Zielerreichung sowie zur Unterstützung individueller Lernprozesse» (Dengel 2018, 23f). Für den Schulkontext ergibt sich die 
Notwendigkeit, Aspekte der digital-vernetzten Welt und medialer Darstellungsformen in enger Anknüpfung an Thematiken der Informationstechnologie und Mediatisierung zu vermitteln. Aus medienpädagogischer Perspektive gilt es, bisherige Medienkompetenzmodelle zu öffnen und mit Aspekten der informationstechnologischen Grundbildung sowie einem gesellschaftskritischen Habitus zu verbinden. Hierzu gibt es unterschiedliche Vorschläge. Bellinger und Kartoglu $(2020,215 \mathrm{ff})$ schlagen etwa eine Orientierung am Konzept der «Digital Liberal Arts Education» vor, in dessen Zentrum ein interdisziplinärer Zugang auf Ausbildung zentraler Kulturtechniken und hier im Speziellen eines Lebens in einer digital-vernetzten Lebenswelt gelegt wird.

Eine Verzahnung von Medienbildung und Informatischer Bildung beschreiben auch Schmid und Gärtig-Daugs $(2018,78)$ in ihrem Ansatz der «Elementarinformatik». Sie betonen zwar ebenso die disziplinäre Trennung von Informatik, Medienpädagogik und Schulpädagogik, zeigen aber gleichzeitig auf, dass in der schulischen Praxis «Mediennutzung und Vermittlung von Informatikkonzepten wechselseitig aufeinander bezogen werden sollten». Wesentlich dabei sei, die originären Ziele der Medienbildung und der Informatischen Bildung beizubehalten. Ziel der Medienbildung bleibt die Ermöglichung eines kritischen, selbst-reflexiven, kreativ-gestalterischen und sicheren Medienhandelns. Hinzu kommen Anliegen der Informatischen Bildung im Sinne der «Vermittlung grundlegender Funktionen und Wirkungsweisen digitaler Technologien», damit diese wiederum kreativ mitgestaltet und kritisch betrachtet werden können (Schmid und Gärtig-Daugs 2018, 81). Kritisch sehen Schmid und Gärtig-Daugs $(2018,86)$ aber die von digitalen Medien losgelöste Vermittlung von Informatikkonzepten. Würde die Vermittlung grundlegender informatischer Prinzipien allzu ausführlich ohne Verknüpfung mit den Besonderheiten digitaler Medien erfolgen, laufe sie Gefahr, dass die Verknüpfungsleistung informatischer Kompetenzen mit Kompetenzen des sicheren und reflexiven Umgangs mit digitalen Medien und der konkreten Lebenswelt unter dem Vorzeichen der Digitalität erschwert wird. Dann werde Informatik zu einem rein analytischen, logischen Fach mit geringen Anknüpfungsmöglichkeiten für die Nutzung digitaler Medien und zur Lebenswelt, also für ein altersspezifisches Medienhandeln.

Die konzeptuelle Verbindung von Aspekten der Medienbildung mit der Informatischen Bildung erfolgt hier mit dem Ziel, aus Perspektive der Informationstechnologie «erste logische und algorithmische Konzepte der Informatik mit anschaulichen, begreifbaren Materialien» sowie "erste Erfahrungen in der Programmierung von einfachen Algorithmen» zu vermitteln. Orientiert an der Medienpädagogik zielen Schmid und Gärtig-Daugs darauf ab, die «Medienkompetenz durch Nutzung verschiedener Anwendungsprogramme» zu fördern, Möglichkeiten der kreativen Mediennutzung aufzuzeigen und einen «kritischen Umgang mit digitalen Medien durch Unterfütterung der Mediennutzung mit informatischen Konzepten» sowie eine «hinterfragenden Grundhaltung» zu fördern. 
Aus Perspektive der Schulpädagogik geht es um die «Integration von Informatik als Querschnittsdisziplin in verschiedene, vor allem auch nicht mathematischnaturwissenschaftliche Unterrichtsfächer», um die «Vermittlung von anknüpfbarem Wissen und systematische Vertiefung von Informatikkonzepten in Form eines Spiralcurriculums», um die "Sensibilisierung und Befähigung von Lehrkräften im Elementar- und Primärbereich für pädagogisch sinnvolle Einsatzmöglichkeiten von digitalen Medien unter Einbeziehung der zugrunde liegenden Informatikkonzepte» (Schmid und Gärtig-Daugs 2018, 99). Dabei sei das konsequente Aufeinanderbeziehen von Informatik, Medienpädagogik und Schulpädagogik dringend notwendig. Denn es braucht die Nutzung digitaler Medien, um die Vermittlung von Informatikkonzepten von einer abstrakten zu einer anschaulich-praktischen Ebene zu heben. Und umgekehrt braucht es Konzepte der Medienbildung, damit sich Kinder und Jugendliche nicht nur als Medienkonsumentinnen und -konsumenten verstehen, sondern als an der digital-vernetzen Medienlandschaft mit einem kritisch, reflexiven Zugang als aktiv teilhabend und mitgestaltende Individuen.

Schmid und Gärtig-Daugs $(2018,78)$ schlagen daher eine Vermittlung von Informatik- und Medienbildungskonzepten aus drei Perspektiven vor: So bräuchte es zum Ersten (1) stets den Bezug «zur Lebenswirklichkeit der Kinder», zum Zweiten (2) einen Zugang des «spielerisch-entdeckende[n] Lernens abstrakter Konzepte durch «begreifbares) Material und ‘Wiedererkennen` der gelernten Konzepte bei der Nutzung digitaler Medien» sowie zum Dritten (3) einen integrativen Ansatz, der die Themen der Informatik und Medienbildung mit anderen Fächern sinnvoll verknüpft (2018, 78).

Dabei orientieren sie $(2018,79 f)$ sich an sogenannten «elementarinformatischen Lerneinheiten», wobei sie die «Elementarinformatik» als «Querschnittsdisziplin» verstehen und darunter eine «spielerische Vermittlung von grundlegenden Informatikkonzepten wie etwa digitale Repräsentation sowie einfache Algorithmen in Zusammenhang mit der Nutzung von Computermedien» verstehen. Ausgehend von der Frage «Wie funktioniert das?» sei das übergeordnete Ziel jeder Lerneinheit, Kinder zum forschenden, entdeckenden Lernen anzuleiten. Lehrpersonen begleiten Schülerinnen und Schüler bei der Suche nach Antworten und können dafür Sorge tragen, dass die Antworten - wie in der Lebenswelt - aus dem Bereich der Informationstechnologie kommen, mit eher sozial-kritischen oder philosophischeren Fragen verbunden sind und in das individuelle Medienhandeln übergeführt werden können. Orientiert an kognitiven Lerntheorien formulieren Schmid und Gärtig-Daugs $(2018,82)$ die Forderung nach der Anregung der Kinder zu Warum-Fragen, zu Wie-funktioniert-dasFragen und zu Fragen der Verbesserung («Wie kann ich das besser machen?») und stellen diesen Fragemodus in das Zentrum ihrer elementarinformatischen Bemühungen. Dadurch würde eine Haltung des «Das-macht-man-halt-so» sowie eine schwerpunktmässig konsumierende Haltung von Kindern, Jugendlichen und Erwachsenen 
erschwert $(2018,87)$. Es geht vordergründig darum, ein «grundlegende[s] Verständnis für die informatischen Konzepte, die hinter der 〈Blackbox) Computer liegen» zu vermitteln. Dadurch findet auch eine Art Entmystifizierung der digitalen Medien statt, welche eine reflektierte und selbstkritische Medienaneignung und ein Medienhandeln möglich machen.

Ein didaktisches Prinzip, welches einer eher praxis- und lebensweltfernen Informatik entgegenwirken soll, ist «Computational Thinking». Jeanette Wing verwendete diesen Begriff erstmals (2006) und zeigte in ihren Ausführungen die oft eingeschränkte Sichtweise der Inhalte von Informatischer Bildung auf: Es geht dabei nicht bloss um das Beherrschen von Programmierfähigkeiten, sondern (auch) um analytisches Denken, Abstraktionsfähigkeit, Musterkennung und Problemlösefähigkeit. Mit dieser offeneren Tätigkeitsbeschreibung auf verschiedenen Abstraktionsebenen werden die ergebnisorientierten, unreflektierten, Programm-schreibenden Coderinnen und Coder zu prozessorientierten, reflektierten, Problem-lösenden Makerinnen und Maker.

Die Konzepte um Computational Thinking schliessen an diese Beschreibung an und werden im Fachdiskurs als eine Art des Denkens und Problemlösens in einer digital-vernetzten Welt diskutiert. Doch eine eindeutige Definition der wesentlichen Inhalte von Computational Thinking ist bisher in der Forschungslandschaft nicht aufzufinden. Die Beschreibungen reichen von einem eher engen Verständnis, welches in der Entwicklungsumgebung von Computerprogrammen verortet ist, bis hin zu einem eher breiten Verständnis von Computational Thinking, welches eine wesentliche Kulturtechnik in der digital-vernetzten Welt darstellt und somit über allgemeinbildende Bildungspläne in der Schule Abbildung finden soll. Computational Thinking ist nach dieser Auffassung der Informatischen Bildung inhärent und soll - wiederum als neue Kulturtechnik - jeder und jedem gleichermassen ermöglicht werden.

Ein engeres Verständnis von Computational Thinking begründet sich darin, dass in der Schulpraxis der Zugang zu informatischem Denken oft in (idealerweise beziehungsreichen) Kontexten passiert, die ihrerseits am Beginn technikorientiert und coding-bezogen sind. Das angestrebte Ziel eines breiteren Verständnisses jenseits von diesem legitimen Zugang wird dabei in der Praxis aber leider oftmals ausgeblendet, da der Übersetzungs- und Arbeitsaufwand zum Entwickeln von Aufgabenstellungen zu beziehungsreichen Kontexten für Lehrerinnen und Lehrer meist zu hoch ist. Somit besteht die Befürchtung, dass - neben der Beschränkung auf den Erwerb von Programmierkenntnissen - die Kompetenzen schrittweise mehr oder weniger theoretisch abgearbeitet werden (Steiner und Himpsl-Gutermann 2020). Mit dieser Beschränkung umfasst Informatische Grundbildung weniger lebensweltnahe Inhalte und erschwert die Entwicklung situativ anwendbarer Problemlösefähigkeiten: 
«While computational thinking is mostly referred to as the computer science approach to problem-solving through abstraction, the use of computational thinking in other disciplines can promote creativity (Bennett, Koh \& Repenning, 2011) and innovation through abstractly conceptualizing the question or challenge. The more frequently students use computational thinking, the better they will become at finding alternative, unique and inventive solutions to complex problems in various domains.» (Ioannidou et al. 2011, 5)

Ioannidou et al. hüten sich dabei vor einer exakten Beschreibung von Computational Thinking und nennen stattdessen Muster («Computational Thinking Patterns»), die sie in Phänomenen und Prozessen in der digitalisierten Welt identifizieren. Auf dieser Grundlage entwickeln und evaluieren sie Lernumgebungen, die einen motivierenden, spielerischen Zugang zum Aufbau von Computational Thinking ermöglichen.

Computational Thinking eröffnet in der pädagogischen Praxis vielfältige Möglichkeiten, um beispielsweise Mediengestaltung aktiv zu erleben oder Phänomene der digital-vernetzten Welt zu verstehen. Somit ist es verständlich, dass Computational Thinking Einzug in Bildungspläne gefunden hat; auch in Österreich. Dabei sind Ansätze einer Verknüpfung von Medienbildung und Informatischer Bildung ersichtlich, die bei einem kritischen Blick allerdings weitere Bemühungen nahelegen.

\section{Ein kritischer Blick auf Implementierung und Inhalte der Verbindlichen Übung «Digitale Grundbildung»}

Im Rahmen der Verbindlichen Übung «Digitale Grundbildung» erhalten Schülerinnen und Schüler in Österreich seit dem Schuljahr 2018/19 an Mittelschulen und an Allgemeinbildenden Höheren Schulen der Unterstufe im Laufe von vier Schuljahren zwischen zwei und vier Wochenstunden eine Grundausbildung zu Themen der Medienbildung und Informatischen Bildung. Bildungs- und Lehraufgabe umfassen laut zugehörigem Lehrplan digitale Kompetenz, Medienkompetenz sowie politische Kompetenzen und intendieren damit die Vermittlung der zunehmenden «Bedeutung von Medien und der über Medien vermittelten Wirklichkeit für die Gesellschaft» (BKA 2018, 14).

Schulen können autonom entscheiden, ob sie diese Grundausbildung als eigenes Fach und/oder fächerintegrativ mit anderen Schulfächern umsetzen. Ein Blick in die pädagogische Ausgestaltung zeigt dabei allerdings, dass die Umsetzung meist integrativ mit anderen Fächern erfolgt. Das könnte unter anderem daran liegen, dass den Schulen für die Umsetzung der Digitalen Grundbildung keine zusätzlichen Ressourcen zur Verfügung gestellt werden und ausserdem keine speziell ausgebildeten Lehrkräfte eingesetzt werden müssen (Grünberger et al. 2021, 932). Ebenso ist die Aus-, Fort- und Weiterbildung der Lehrkräfte in diesem Kontext bei weitem nicht 
einheitlich und an manchen Hochschulen erst in Entwicklung. Mehrere Spannungsfelder in der schulischen Umsetzung sind somit durchaus ersichtlich, nicht zuletzt weil Aushandlungsprozesse an die Schule verlagert sind. Neben der fachlichen Trägerschaft (ein Fach vs. viele Fächer) und der Qualifikation der Lehrkräfte (spezielle Ausbildung vs. Fachexpertise) sind zu verhandelnde Punkte vor allem die curriculare Verbindlichkeit (offenes vs. geschlossenes Curriculum) und die Leistungsbeurteilung (zensurenfrei vs. benotet) (ebd, 937).

Im verordneten Lehrplan werden acht Kompetenzbereiche genannt (BKA 2018, 16ff):

- Gesellschaftliche Aspekte von Medienwandel und Digitalisierung

- Informations-, Daten- und Medienkompetenz

- Betriebssysteme und Standard-Anwendungen

- Mediengestaltung

- Digitale Kommunikation und Social Media

- Sicherheit

- Technische Problemlösung

- Computational Thinking

Mit einem Blick auf diese Kompetenzbereiche wird eine breite medien- und informatiksensible Bildung angebahnt und der gesellschaftlichen Notwendigkeit der Verzahnung der Informatischen Bildung mit der Medienbildung entgegengetreten; auf Mikroebene werden überlappende Bereiche allerdings nur schwer gefunden, wie ein genauerer Blick beispielsweise auf den Kompetenzbereich «Computational Thinking» zeigt. Im Lehrplan werden für Computational Thinking die beiden Teilbereiche «Mit Algorithmen arbeiten» und "Kreative Nutzung von Programmiersprache» angeführt. In welcher Weise Aspekte der Medienbildung hierbei abgebildet werden sollen, wird nicht erwähnt. Somit liegt erneut eine enge Interpretation von Computational Thinking nahe, welche Computational Thinking mit dem Erwerb von Programmierkenntnissen gleichsetzt. Die zweigleisige Koexistenz von Medienpädagogik und Informatik als Bezugsdisziplinen von Medienbildung und Informatischer Bildung ist somit weiterhin wahrnehmbar (Rummler et al. 2016).

Indem Computational Thinking im Lehrplan zur Verbindlichen Übung «Digitale Grundbildung» genannt wird, folgt Österreich dem internationalen Trend, Computational Thinking in Bildungsplänen abzubilden. Im Unterschied zu anderen Kompetenzmodellen, wie beispielsweise «DigComp 2.1» (Carretero, Vuorikari, und Punie 2017), werden dabei aber im österreichischen Lehrplan mögliche Verzahnungen der Medienbildung mit der Informatischen Bildung ausgespart. 


\section{Conclusio}

Wie kann nun das Beziehungsverhältnis von Medienbildung und Informatischer Bildung näher spezifiziert werden? Und wie können beide Disziplinen in der pädagogischen Praxis abgebildet werden um den Anforderungen einer Mediatisierung der Lebenswelt gerecht zu werden? - Medienbildung bietet eine weitreichende «Reflexionsebene» sowie «Anwendungskenntnisse», Informatik liefert das für die «Anwendungskenntnisse und Medienbildung notwendige Grundlagenwissen» (Döbeli Honegger 2017, 78). Doch bleiben wir einem, den Traditionen einer disziplinären Verortung verpflichteten Zugang treu, kann es keine systematische Verbindung der beiden Diskurse geben. Daraus folgt, dass auch jeder Auftrag der transdisziplinären Zusammenführung der Positionen für eine pädagogische Praxis - wie mancherorts gefordert - nicht nur erschwert wird, sondern sogar unmöglich scheint. Doch angesichts weitreichender gesellschaftlicher Herausforderungen, denen nur im transdisziplinären Zusammenspiel begegnet werden kann, ist es gewissermassen notwendig, einen Zugang zueinander zu finden und zusammenzuarbeiten. Ein solches transdisziplinäres Denken kann und sollte bereits in der Elementar-/Primarstufe adressiert und in der Sekundarstufe weiterentwickelt werden. Für den Schulkontext gilt es generell, die Verbindung einer informationstechnologischen, einer sozialen und einer individuell-reflexiven Ebene im Medienhandeln und der kritischen Reflexion darüber zu leben sowie interaktive, konstruktive Lehr-Lernkonzepte, wie sie durch digitale Medien möglich werden, zu entwickeln und umzusetzen (Schmid und Gärtig-Daugs 2018, 79). Nicht nur in österreichischen Lehrplänen ist dieses Desiderat bereits als Bildungs- und Lehraufgabe verankert. Im Unterschied zu Kompetenzmodellen von anderen Ländern werden aber im österreichischen Lehrplan für die Verbindliche Übung «Digitale Grundbildung» mögliche Verzahnungen der Medienbildung mit der Informatischen Bildung ausgespart, sodass weitere politische Massnahmen notwendig werden. Angesichts der weitreichenden Herausforderungen vor denen unsere Gesellschaft steht, oder in den Worten Klafkis (1998): Angesichts der zu lösenden «epochaltypischen Schlüsselprobleme» wie beispielsweise Friedensfragen, Umweltprobleme, Gefahren und Möglichkeiten der neuen technischen Steuerungs-, Informations-, und Kommunikationsmedien usw. scheint das Aufeinander-Beziehen oder die Verzahnung der Informatischen Bildung mit der Medienbildung nicht nur sinnvoll, sondern notwendig. In diesem Sinne kann die Medienbildung eine kritischreflexive Medienaneignung sowie den weitreichenden Blick auf die Mediatisierung unserer Gesellschaft und die Informatik ein grundlegendes Verstehen der Funktion informatischer Systeme sowie erprobte Problemlösungsstrategien fördern. So wird es möglich, Medien, Informationen und Phänomenen unserer Umwelt kritisch und mitgestaltend zu begegnen, um damit auch aktuelle gesellschaftliche Herausforderungen unserer Welt bewältigen zu können. 


\section{Literatur}

Bellinger, Franziska, und Cansu Kartoglu. 2020. «Digital Liberal Arts in der Primarstufe. Medienbildung und informatische Bildung im Grundschulalter». In Digitale Bildung im Grundschulalter. Grundsatzfragen zum Primat des Pädagogischen, herausgegeben von Mareike Thumel, Rudolf Kammerl, und Thomas Irion, 215-229. München: Kopaed Verlag. https:// doi.org/10.25593/978-3-86736-543-7.

Bettinger, Patrick, und Sandra Aßmann. 2017. «Das diskursive Feld um Mediatisierung und Mediensozialisation. Eine Analyse deutschsprachiger Fachzeitschriften». In Mediatisierung und Mediensozialisation: Prozesse - Räume - Praktiken, herausgegeben von Dagmar Hoffmann, Friedrich Krotz, und Wolfgang Reißmann, 79-100. Medien • Kultur • Kommunikation. Wiesbaden: Springer Fachmedien. https://doi.org/10.1007/978-3-658-14937-6_5.

BKA (Bundeskanzleramt der Republik Österreich) Rechtsinformationssystem des Bundes (RIS), Hrsg. 2018. Änderung der Verordnung über die Lehrpläne der Neuen Mittelschulen sowie der Verordnung über die Lehrpläne der allgemeinbildenden höheren Schulen. https://www.ris.bka.gv.at/Dokumente/BgblAuth/BGBLA_2018_II_71/BGBLA_2018_II_71.pdf.

Brinda, Torsten, Ira Diethelm, Rainer Gemulla, Ralf Romeike, Johannes Schöning, Carsten Schulte, et al. 2016. "Dagstuhl-Erklärung: Bildung in der digitalen vernetzten Welt». https://gi.de/themen/beitrag/dagstuhl-erklaerung-bildung-in-der-digital-vernetzten-welt.

Brinda, Torsten, Niels Brüggen, Ira Diethelm, Thomas Knaus, Sven Kommer, Christine Kopf, Petra Missomelius, Rainer Leschke, Friederike Tilemann, und Andreas Weich. 2019. «Frankfurt-Dreieck zur Bildung in der digital vernetzten Welt. Ein interdisziplinäres Modell». Gesellschaft für Informatik. https://dagstuhl.gi.de/fileadmin/Gl/Allgemein/PDF/FrankfurtDreieck-zur-Bildung-in-der-digitalen-Welt.pdf.

Brinda, Torsten. 2016. Stellungnahme zum KMK-Strategiepaper «Bildung in der digitalen Welt». https://fb-iad.gi.de/fileadmin/FB/IAD/Dokumente/gi-fbiad-stellungnahme-kmk-strategiedigitale-bildung.pdf.

Bundesministerium für Digitalisierung und Wirtschaftsstandort. 2016. «Die digitale Strategie der österreichischen Bundesregierung». Wien: Österreichisches Bundesministerium für Digitalisierung und Wirtschaftsstandort. https://www.digitalroadmap.gv.at.

Carretero, Stephanie, Riina Vuorikari, und Yves Punie. 2017. DigComp 2.1: The Digital Competence Framework for Citizens. With Eight Proficiency Levels and Examples of Use. Luxembourg. https://doi.org/10.2760/38842.

Dengel, Andreas. 2018. «Digitale Bildung: ein interdisziplinäres Verständnis zwischen Medienpädagogik und Informatik». MedienPädagogik. Zeitschrift für Theorie und Praxis der Medienbildung 33 (Didaktik der Informatik):11-26. https://doi.org/10.21240/mpaed/33/2018.10.30.X.

Deutsche Kultusministerkonferenz. 2016. «Bildung in der digitalen Welt. Strategie der Kultusministerkonferenz». Berlin: Sekretariat der Kultusministerkonferenz. http://www.kmk.org/ fileadmin/Dateien/pdf/PresseUndAktuelles/2016/Bildung_digitale_Welt_Webversion.pdf.

Döbeli Honegger, Beat. 2017. Mehr als 0 und 1. Schule in einer digitalisierten Welt. Bern: hep Verlag. 
Faßler, Manfred. 2009. Nach der Gesellschaft: infogene Welten, anthropologische Zukünfte. München: Wilhelm Fink.

Fromme, Johannes, und Benjamin Jörissen. 2010. «Medienbildung und Medienkompetenz. Berührungspunkte und Differenzen nicht ineinander überführbarer Konzepte». medien + erziehung 54 (5), 46-54.

Gesellschaft für Medienpädagogik und Kommunikationskultur. 2016. Stellungnahme der Gesellschaft für Medienpädagogik und Kommunikationskultur (GMK) zum Strategie-Papier der KMK «Bildung in der digitalen Welt». 27. April. http://www.gmk-net.de/fileadmin/pdf/GMKStellungnahme_zum_KMK-Strategie-Entwurf.pdf.

Grünberger, Nina. 2017. «Medienpädagogik?! Ein Suchen nach einer kontemporären Disziplin». In Medienpädagogik. Eine Standortbestimmung, herausgegeben von Anja HartungGriemberg und Christine Trültzsch-Wijnen, 103-14. Baden-Baden: Nomos-Verlag. https:// doi.org/10.5771/9783845279718-103.

Grünberger, Nina, Thomas Narosy, und Michael Schratz. 2021. «Der Lehrplan „Digitale Grundbildung“ für alle. „Policy Enactment“ in der Digitalität». In Handbuch Lernen mit digitalen Medien, herausgegeben von Gerold Brägger und Hans-Günter Rolff, 925-945. Weinheim u.a.: Beltz.

Hüther, Jürgen, und Bernd Podehl. 2005. «Geschichte der Medienpädagogik». In Grundbegriffe der Medienpädagogik, herausgegeben von Bernd Schorb und Jürgen Hüther, 4. vollständig neu konzipierte Auflage, 116-27. München: Kopaed Verlag.

Ioannidou, Andri, Bennett, Vicki, Repenning, Alexander, Koh, Kyu Han, und Basawapatna, Ashok. 2011. Computational Thinking Patterns. Paper presented at the Annual Meeting of the American Educational Research Association, Online Submission, New Orleans, LA. https:// eric.ed.gov/?id=ED520742.

Iske, Stefan. 2015. «Medienbildung». In Medienpädagogik - ein Überblick, herausgegeben von Friederike von Gross, Dorothee M. Meister und Uwe Sander, 247-73. Weinheim u.a.: Beltz Juventa.

Klafki, Wolfgang. 1996. «Grundzüge eines neuen Allgemeinbildungskonzepts. Im Zentrum: Epochaltypische Schlüsselprobleme». In Neue Studien zur Bildungstheorie und DidaktikZeitgemäße Allgemeinbildung und kritisch-konstruktive Didaktik, 5. unveränderte Aufl., 4381. Weinheim u.a.: Beltz (Reihe Pädagogik).

Klafki, Wolfgang. 1998. «Schlüsselprobleme der modernen Welt und die Aufgaben der Schule - Grundlinien einer neuen Allgemeinbildungskonzeption in internationaler/interkultureller Perspektive». In Pluralität und Bildung, herausgegeben von Ingrid Gogolin, Marianne Krüger-Potratz, und Meinert A. Meyer, 235-49. Schriften der Deutschen Gesellschaft für Erziehungswissenschaften (DGfE). Opladen: Leske + Budrich. https://doi.org/10.1007/9783-663-11056-9_14.

Krotz, Friedrich. 2001. Die Mediatisierung kommunikativen Handelns: der Wandel von Alltag und sozialen Beziehungen, Kultur und Gesellschaft durch die Medien. 1. Aufl. Wiesbaden: WestdtVerl. https://doi.org/10.1007/978-3-322-90411-9. 
Lyotard, Jean-François. 1979. Das postmoderne Wissen: Ein Bericht. Übersetzt von Otto Pfersmann. 7., ü.a. Aufl. Wien: Passagen Verlag (2012).

McLuhan, Marshall. 1962. Die Gutenberg-Galaxis. Das Ende des Buchzeitalters. Düsseldorf und Wien: Econ-Verlag (1968).

Meder, Norbert. 2004. Der Sprachspieler: Der postmoderne Mensch oder das Bildungsideal im Zeitalter der neuen Technologien. 2. wesentl. erw. Aufl. Würzburg: Königshausen und Neumann.

Moser, Heinz. 2006. Einführung in die Medienpädagogik. Aufwachsen im Medienzeitalter. 4. ü.a., aktualisierte Auflage. Wiesbaden: VS Verlag für Sozialwissenschaften. https://doi. org/10.1007/978-3-531-90589-1.

Rummler, Klaus, Beat Döbeli Honegger, Heinz Moser, und Horst Niesyto. 2016. «Editorial: Medienbildung und informatische Bildung - quo vadis?». MedienPädagogik: Zeitschrift für Theorie und Praxis der Medienbildung. 25 (Computer Science Education), 1-6. https://doi. org/10.21240/mpaed/25/2016.10.24.X.

Schiefner-Rohs, Mandy. 2017. «Medienbildung in der Schule. Blinde Flecken und Spannungsfelder in einer Kultur der Digitalität». MedienPädagogik. Zeitschrift für Theorie und Praxis der Medienbildung 27 (Spannungsfelder und blinde Flecken): 153-72. https://doi. org/10.21240/mpaed/27/2017.10.15.X.

Schmid, Ute, und Anja Gärtig-Daugs. 2018. «Notwendigkeit der Integration elementarinformatischer Lerneinheiten in den Vor- und Grundschulunterricht». MedienPädagogik. Zeitschrift für Theorie und Praxis der Medienbildung 31 (‘Digitale Bildung〉): 78-106. https://doi. org/10.21240/mpaed/31/2018.03.29.X.

Schorb, Bernd. 2011. "Zur Theorie der Medienpädagogik». In Medienbildung und Medienkompetenz: Beiträge zu Schlüsselbegriffen der Medienpädagogik, herausgegeben von Heinz Moser, Petra Grell, und Horst Niesyto, 81-94. München: Kopaed Verlag. https://doi. org/10.21240/mpaed/20/2011.09.14.X.

Steiner, Michael, und Himpsl-Gutermann, Klaus. 2020. «Computational Thinking und Kontextorientierung». Medienimpulse. 58 (1). https://doi.org/10.21243/mi-01-20-21.

Trültzsch-Wijnen, Christine, Hrsg. 2017. Medienpädagogik. Eine Standortbestimmung. Bd. 1. Medienpädagogik | Media Education. Baden-Baden: Nomos. https://doi. org/10.5771/9783845279718.

von Weizsäcker, Ernst Ulrich, und Anders Wijkman. 2018. Wir sind dran. Club of Rome: Der große Bericht. Gütersloher Verlagshaus.

WBGU - Wissenschaftlicher Beitrag der Bundesregierung Globale Umweltveränderungen. 2019. «Unsere gemeinsame digitale Zukunft». Berlin: WBGU. https://www.wbgu.de/de/publikationen/publikation/unsere-gemeinsame-digitale-zukunft\#sektion-downloads.

Wing, Jeannette M. 2006. «Computational Thinking». Communications of the ACM. 49 (3), 33-35. https://doi.org/10.1145/1118178.1118215. 